\title{
Clostridium difficile infection and antibiotic-associated diarrhoea
}

\author{
Authors: Benjamin H Mullish ${ }^{A}$ and Horace RT Williams ${ }^{B}$
}

\begin{abstract}
Antibiotic-associated diarrhoea is among the most common adverse events related to antibiotic use. Most cases are mild, but Clostridium difficile infection causes a spectrum of disease, ranging from occasional diarrhoea to colitis, toxic megacolon, and potentially death. Recent developments in our understanding of the biology of the gut microbiota have provided new insights into the pathogenesis of these conditions, and have revealed a role for manipulation of the gut microbiota as a novel therapeutic approach. This review will give an overview of the assessment of these conditions, before focusing on the rapidly developing area of their treatment.
\end{abstract}

\section{Introduction}

In addition to being a potential reservoir for pathogenic bacteria the gut also has an extensive ecosystem of microorganisms $\left(\sim 10^{11}\right.$ bacteria per gram of intestinal content) with no overt pathogenicity. It is now appreciated that this ecosystem (often called the 'gut microbiota') performs roles that are essential for the maintenance of host health, including short chain fatty acid and bile acid metabolism. ${ }^{1}$ Antibiotic-associated diarrhoea (AAD) is now understood to represent an imbalance of the gut microbiota resulting from antibiotic use, with several mechanisms appearing to contribute to the disease process.

Most cases of AAD are mild and self-limiting, and are associated with negative stool culture results. Given the widespread use of antibiotics, it is not surprising that this condition is so common, affecting $5-39 \%$ of people treated with antibiotics. ${ }^{2}$ One key mechanism of AAD appears to be changes in the gut microbiota that cause decreased short-chain fatty acid absorption, resulting in osmotic diarrhoea. ${ }^{3}$

One particular form of AAD - Clostridium difficile infection (CDI) - can cause more severe gastrointestinal disease. Clostridium difficile (now also referred to as Clostridioides difficile) is a Grampositive, spore-forming anaerobic bacillus. Spores of $C$ difficile can survive for long periods on inanimate objects (resisting heat, acid and antibiotics), a major reason why this bacterium can cause such problems within healthcare settings. Clostridium difficile is spread

Authors: ${ }^{A}$ specialty registrar and MRC clinical research training fellow, St Mary's Hospital Campus, Imperial College London, London, UK; B consultant gastroenterologist and honorary senior lecturer, St Mary's Hospital Campus, Imperial College London, London, UK via the faeco-oral route, and causes disease in humans through the production of two protein exotoxins (toxin A and toxin B), which are cytotoxic to colonic epithelial cells. ${ }^{4}$ The host's adaptive immune response following exposure to $C$ difficile also appears to be important in determining the severity of the disease, with high IgG antitoxin level production being protective. ${ }^{4}$ Antibiotic use is the major risk factor for CDI, causing antibiotic-related loss of gut microbial communities that protect against gut infection, thereby facilitating the germination and vegetative growth of the organism when it enters the gut of vulnerable people. Risk factors for CDI are summarised in Table 1. The range of clinical disease that can occur in CDI is wide: diarrhoea and fever occur in almost all cases, but the most severe cases are characterised by colitis, toxic megacolon (dilatation of the colon, with the risk of perforation), multi-organ failure, or even death.

\section{Key points}

Antibiotic use may result in diarrhoea through different mechanisms, including: osmotic diarrhoea (through the loss of gut bacteria that absorb short-chain fatty acids); and colonisation and overgrowth of toxin-secreting Clostridium difficile

Typical laboratory tests for the presence of $C$ difficile do not have high sensitivity and specificity in isolation, so a combination of tests should be used when making the diagnosis of CDI

CDI has recently become harder to treat with conventional antibiotics for several reasons, including increased treatment failure with metronidazole, rising rates of $\mathrm{CDI}$ recurrence, and the emergence of hypervirulent strains of C difficile (eg NAP1/027)

Fidaxomicin is a new antibiotic that has considerable efficacy in treating recurrent $\mathrm{CDI}$, though there is uncertainty as to its use in particular situations, including treatment of the NAP1/027 strain

Randomised clinical trial evidence demonstrates that faecal microbiota transplant (FMT) is more effective than vancomycin for the treatment of recurrent or refractory CDI, and this treatment is now approved in guidelines

KEYWORDS: Clostridium difficile, diarrhoea, antibiotics, faecal microbiota transplant, microbiota 
Table 1. Risk factors for Clostridium difficile infection

\begin{tabular}{|c|c|}
\hline Risk factor & Details \\
\hline Antibiotics & $\begin{array}{l}\text { Almost all antibiotics can increase } \\
\text { vulnerability to Clostridium difficile } \\
\text { infection (CDI), but cephalosporins, } \\
\text { fluoroquinolones, clindamycin and certain } \\
\text { penicillins (eg co-amoxiclav) increase risk } \\
\text { to the greatest extent }\end{array}$ \\
\hline $\begin{array}{l}\text { Acid-suppressant } \\
\text { medications }\end{array}$ & $\begin{array}{l}\text { Both proton-pump inhibitors (PPI) and } \mathrm{H}_{2} \\
\text { receptor antagonists appear to increase } \\
\text { risk (risk is increased with PPI especially) }\end{array}$ \\
\hline Age & $\begin{array}{l}\text { Rates are ten-fold higher in those of }>65 \\
\text { years than in the younger population }\end{array}$ \\
\hline \multirow[t]{2}{*}{ Hospitalisation } & $\begin{array}{l}\text { Recent hospitalisation, prolonged } \\
\text { hospitalisation ( }>7 \text { days), and/ } \\
\text { or prolonged antibiotic courses all } \\
\text { increase risk of CDI }\end{array}$ \\
\hline & $\begin{array}{l}\text { Being admitted to a room where the } \\
\text { previous patient had CDI is itself a risk } \\
\text { factor for infection }\end{array}$ \\
\hline Immunosuppres & \\
\hline
\end{tabular}

Rates of CDI (and associated morbidity/mortality) peaked in the UK approximately 10 years ago (with about 70,000 cases in the UK, around 4000 of which were fatal, attributed to CDI in 2007). ${ }^{5}$ Rates have since decreased rapidly. There are currently around 12,000 cases per year in the UK, ${ }^{5}$ with the sharp recent decline in incidence being attributed to infection prevention and control interventions, including antibiotic stewardship. ${ }^{6}$

\section{Diagnosis}

Typical AAD is a clinical diagnosis, based on the relationship between recent antibiotic use and the development of diarrhoea, and on the exclusion of potential alternative causes of diarrhoea.

Diagnosis of CDI may be difficult for several reasons. First, the currently available tests lack ideal sensitivity and specificity. Second, $C$ difficile may either cause asymptomatic colonisation or active gastrointestinal infection, and conventional tests do not clearly differentiate between these two states.

Therefore, guidelines recommend testing for CDI only when a patient has diarrhoea and grounds for suspecting an infective aetiology. ${ }^{7}$ Laboratory tests for CDI are summarised in Table 2

Several other investigations may also be useful in the assessment of people with CDI. Leucocytosis (particularly neutrophilia) is common, and may be very marked. Abdominal radiograph is the preferred test for toxic megacolon, whereas abdominal $\mathrm{CT}$ is sensitive for the presence of CDI-related colitis. Lower gastrointestinal endoscopy may show only oedema or erythema, or may show the classical finding of pseudomembranes, ie raised yellowish plaques that may be intermittently scattered throughout affected colonic mucosa (Fig 1).

Where clinicians have a strong clinical suspicion of CDI, empirical therapy should be considered regardless of the laboratory result;
Table 2. Tests for Clostridium difficile infection

\section{Test}

GDH EIA

Toxin A and This test has high specificity but variable degrees B EIA of sensitivity

Toxin A and This PCR test is specific for genes encoding toxins B PCR that are produced by $C$ difficile, but a positive result does not help to distinguish active toxin production from asymptomatic carriage of toxigenic $C$ difficile

Cell culture This is still widely recognised as the reference cytotoxicity standard test, although it is rarely performed in assay most clinical laboratories
Given the variability of the sensitivity and specificity of these tests, a two-stage testing algorithm is widely applied. In the UK, toxin A/B enzyme immunoassay (EIA) testing is often used after an initial glutamate dehydrogenase (GDH) or polymerase chain reaction (PCR) screen

this is because with a high pre-test probability, the negative predictive value of the tests that are currently available is insufficient to exclude disease.

\section{Treatment}

In all cases of $A A D$, initial care includes the removal of precipitating factors (ie cessation of antibiotics). This will resolve the majority of cases of typical AAD (and some cases of CDI), but most cases of CDI will require more specific intervention. In treating CDI, a severity assessment is important to establish the most appropriate treatment. The assessment described in

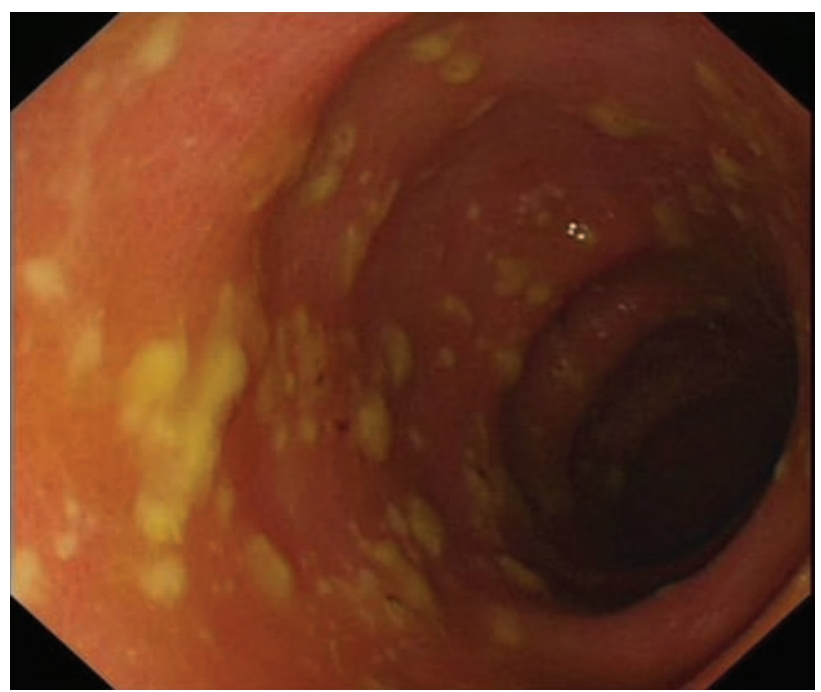

Fig 1. Pseudomembranous colitis. 
the current Public Health England (PHE) guidelines ${ }^{8}$ is the most commonly used version:

$>$ white cell count $>15 \times 10^{9} / \mathrm{L}$

$>$ acutely rising creatinine (eg $>50 \%$ increase above baseline)

$>$ temperature $>38.5^{\circ} \mathrm{C}$

$>$ severe colitis (either from clinical abdominal signs or from radiological investigations).

\section{Conventional treatment of CDI}

Until recently, the antibiotics metronidazole and vancomycin were the only pharmacological options for the treatment of CDI. Metronidazole may be administered orally or intravenously, whereas vancomycin may be given orally or per rectum for this indication. Vancomycin is not used intravenously to treat CDI because it has very limited penetration into the gut mucosa. Intravenous immunoglobulin (IVIg) also has a role in treating severe disease. Until recently, these treatments were effective for most cases of CDI, with surgical intervention (colectomy) reserved for the small number of severe cases that were refractory to medical therapy. ${ }^{8}$

However, over the past decade, CDI has become more difficult to treat with conventional therapies for several reasons. First, the rate of CDI treatment failure with metronidazole is increasing, with rate of non-response now quoted at $>20 \% ;{ }^{9}$ this is one of the reasons why patients with CDI and markers of severity should be treated with vancomycin in preference to metronidazole. ${ }^{8}$ Second, rates of recurrent CDI (ie return of disease within a short period after stopping antibiotic therapy) are also increasing. ${ }^{9}$ Third, hypervirulent strains of $C$ difficile have emerged, particularly the NAP1/027 strain, which is associated with the secretion of large quantities of toxin and poor response to conventional antibiotics. ${ }^{10}$ Novel approaches for therapy for CDI are urgently sought.

\section{New antibiotics: fidaxomicin}

Fidaxomicin is a novel macrocyclic antibiotic, administered orally, with a narrow spectrum of activity. Although fidaxomicin has been shown in clinical trials to have efficacy in treating recurrent $\mathrm{CDI}^{11}$ (and has consequently been added into clinical guidelines ${ }^{8}$ ), there are still a number of concerns regarding its use. These include its apparently limited efficacy against the NAP1/027 strain, the lack of evidence regarding its use in CDI with severe colitis, and its considerable expense (since it is 600 times more expensive than metronidazole.)

\section{Manipulation of the gut microbiota: probiotics and faecal microbiota transplantation}

Given that all forms of AAD represent a perturbation of the gut microbiota, restoration of the usual commensal microbiota is an appealing means of treating the condition.

One approach to achieve this is through the use of probiotics, ie the delivery of live microorganisms into the host (sometimes through a suspension or drink). There are limited data regarding the use of probiotics to treat AAD/CDI, with relevant studies suggesting a possible modest benefit in treating non-severe CDI. However, significant uncertainties still exist in determining the specific organisms that should be included in the formulation, the dose to be used and so on, and probiotics are therefore not recommended at present.
Another approach has been the use of faecal microbiota transplantation (FMT), that is, taking a stool sample from a healthy screened donor (with presumed 'normal' gut microbiota), processing this in a laboratory into a liquidised bacterial suspension, and delivering it into the gut of affected people. A number of case reports and small case series have described this treatment over the past 60 years, but the first major randomised trial investigating its efficacy was carried out in $2013 .^{12}$ In this study, patients with recurrent CDI were randomised to receive either bowel lavage followed by FMT (via a nasoduodenal tube), vancomycin alone, or vancomycin with bowel lavage. The study was stopped early on ethical grounds after a planned interim analysis because $81 \%$ of patients $(n=13 / 16)$ had recovered in the FMT arm, compared to $31 \%(n=4 / 13)$ receiving vancomycin alone, and $23 \%(n=3 / 13)$ receiving vancomycin and bowel lavage. Two of the three patients who did not respond to an initial FMT were successfully treated with a second. There were no major adverse effects. A subsequent randomised trial demonstrated that FMT was superior to vancomycin in treating recurrent CDI when administered colonoscopically. ${ }^{13}$

These and other studies have helped to establish optimal practical aspects related to FMT administration (Table 3). FMT appears generally safe with mild, self-limiting gastrointestinal and constitutional symptoms being the most common complications described. However, there have been a small number of potential transmissions of infection, perforations associated with treatment administration, and even deaths related to its use (eg through aspiration of transplant material). ${ }^{14}$ As a result of these clinical studies (coupled with other studies demonstrating its cost effectiveness), FMT has now been accepted as an approved treatment for recurrent or refractory CDI by $\mathrm{NICE}^{15}$ and in PHE guidelines. ${ }^{8} \mathrm{FMT}$ is now available as treatment for $\mathrm{CDI}$ within a growing number of UK centres, but the expense of establishing and maintaining a service, and ongoing discussions about the regulation and governance of FMT within the UK, have impacted upon more rapid adoption. ${ }^{16}$

Given the obvious potential drawbacks of FMT (including its unpalatable nature and the invasive means of administration), there is great interest in refining FMT from its current state into a more acceptable pill or drink. 'Capsulised' FMT (in which faecal slurry or freeze-dried stool is placed into capsules) is now starting to become available and has recently been demonstrated to be of similar efficacy to colonoscopic FMT in a large randomised trial. $^{17}$

\section{Other novel approaches}

A number of novel approaches for the treatment of CDI are at various stages of development; these are summarised in Table 4.

\section{Conclusions}

$A A D$ and $C D I$ remain a common problems. Many cases are mild, but a significant number - especially of CDI - can be difficult to treat and potentially life-threatening. Developments in our understanding of the importance of the gut microbiota, and the marked efficacy of FMT in clinical trials, have raised the profile of CDI research and the search for more refined, targeted therapies. 
Table 3. Practical aspects of faecal microbiota transplantation (FMT). (Adapted from Mullish et al ${ }^{18}$ and Cammarota et al ${ }^{19}$ )

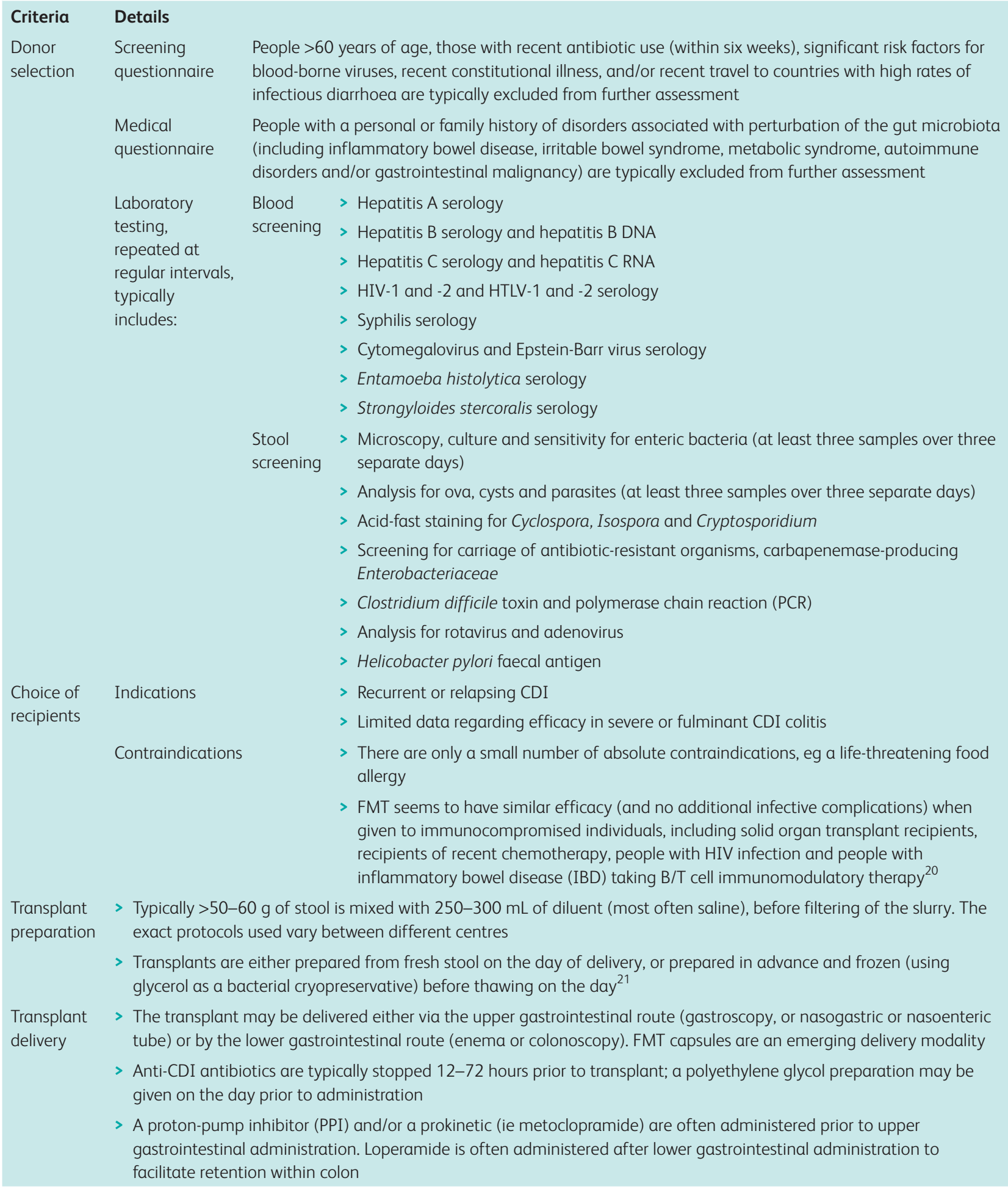


Table 4. Novel approaches for the treatment of Clostridium difficile infection (CDI)

\begin{tabular}{|c|c|c|}
\hline Approach & Details & \\
\hline \multirow[t]{2}{*}{ Antimicrobials } & Antibiotics & A number of novel antibiotics are in clinical trials \\
\hline & Ribaxamase & $\begin{array}{l}\text { This is a beta-lactamase that may be co-administered with systemic broad-spectrum antibiotics, with } \\
\text { the aim of degrading the antibiotics in the gut before they can affect the gut microbiota and create a } \\
\text { propensity to CDI }\end{array}$ \\
\hline \multirow[t]{2}{*}{ Immunisation } & $\begin{array}{l}\text { Passive } \\
\text { immunisation }\end{array}$ & $\begin{array}{l}\text { Co-administration of bezlotoxumab (an anti-toxin B antibody) together with conventional antibiotic } \\
\text { therapy has been shown to reduce the rate of recurrence of CDI compared to antibiotic therapy alone }{ }^{22}\end{array}$ \\
\hline & $\begin{array}{l}\text { Active } \\
\text { immunisation }\end{array}$ & $\begin{array}{l}\text { Anti-toxin vaccines are now in clinical trials. However, early data suggest reduced seroconversion in } \\
\text { older people, those most at risk of CDI }\end{array}$ \\
\hline $\begin{array}{l}\text { Microbiome } \\
\text { manipulation }\end{array}$ & $\begin{array}{l}\text { Non-toxigenic } \\
\text { C difficile }^{23}\end{array}$ & $\begin{array}{l}\text { In a clinical trial, non-toxigenic } C \text { difficile was administered to those with CDI, with the aim of } \\
\text { outcompeting toxigenic } C \text { difficile from its reservoir within the gut. CDI recurrence rates were } 30 \% \text { in } \\
\text { those receiving placebo in comparison with } 11 \% \text { in those receiving non-toxigenic } C \text { difficile }\end{array}$ \\
\hline
\end{tabular}

\section{References}

1 Marchesi JR, Adams DH, Fava F et al. The gut microbiota and host health: a new clinical frontier. Gut 2016:65:330-9.

2 McFarland LV. Epidemiology, risk factors and treatments for antibiotic-associated diarrhea. Dig Dis 16:292-307.

3 Högenauer C, Hammer HF, Krejs G], Reisinger EC. Mechanisms and management of antibiotic-associated diarrhea. Clin Infect Dis 1998:27:702-10.

4 Rupnik M, Wilcox MH, Gerding DN. Clostridium difficile infection: new developments in epidemiology and pathogenesis. Nat Rev Microbiol 2009; 7:526-36.

5 Public Health England. Clostridium difficile: guidance, data and analysis. www.gov.uk/government/collections/clostridium-difficileguidance-data-and-analysis [Accessed 8 April 2018].

6 Aldeyab MA, Kearney MP, Scott MG, et al. An evaluation of the impact of antibiotic stewardship on reducing the use of high-risk antibiotics and its effect on the incidence of Clostridium difficile infection in hospital settings. J Antimicrob Chemother 2012;67:2988-96.

7 Public Health England. Updated guidance on the diagnosis and reporting of Clostridium difficile, 2012. www.gov.uk/government/ uploads/system/uploads/attachment_data/file/215135/dh_133016. pdf [Accessed 18 April 2018].

8 Public Health England. Updated guidance on the management and treatment of Clostridium difficile infection, 2013. www.gov.uk/ government/uploads/system/uploads/attachment_data/file/321891/ Clostridium_difficile_management_and_treatment.pdf [Accessed 18 April 2018]

9 Kelly CP, LaMont JT. Clostridium difficile - more difficult than ever. N Engl J Med 2008:359:193-240.

10 Martin JSH, Monaghan TM, Wilcox MH. Clostridium difficile infection: epidemiology, diagnosis and understanding transmission. Nat Rev Gastroenterol Hepatol 2016;13:206-16.

11 Louie TJ, Miller MA, Mullane KM et al. Fidaxomicin versus vancomycin for Clostridium difficile Infection. N Engl J Med 2011;364:422-31.

12 van Nood E Vrieze A, Nieuwdorp M et al. Duodenal infusion of donor feces for recurrent Clostridium difficile. N Engl J Med 2013;368:407-15.

13 Cammarota G, Masucci L, Ianiro G et al. Randomised clinical trial: faecal microbiota transplantation by colonoscopy vs. vancomycin for the treatment of recurrent Clostridium difficile infection. Aliment Pharmacol Ther 2015;41:835-43.
14 Baxter M, Colville A. Adverse events in faecal microbiota transplant: a review of the literature. J Hosp Infect 2016;92:117-27.

15 National Institute for Health Care Excellence. Faecal microbiota transplant for recurrent Clostridium difficile infection. Interventional procedure guidance, 2014. www.nice.org.uk/guidance/ipg485/ resources/faecal-microbiota-transplant-for-recurrent-clostridiumdifficile-infection-1899869993554885 [Accessed 20 March, 2017].

16 Mullish BH, Williams HRT. Obstacles to establishing an NHS faecal transplant programme. BMJ 2015;351:h6043.

17 Kao D, Roach B, Silva M et al. Effect of oral capsule-vs colonoscopydelivered fecal microbiota transplantation on recurrent Clostridum difficile infection. JAMA 2017;318:1985-93.

18 Mullish BH, Marchesi JR, Thursz MR, Williams HRT. Microbiome manipulation with faecal microbiome transplantation as a therapeutic strategy in Clostridium difficile infection. QJM 2015;108:355-9.

19 Cammarota $\mathrm{G}$, Ianiro G, Tilg $\mathrm{H}$ et al. European consensus conference on faecal microbiota transplantation in clinical practice. Gut 2017:66:569-80.

20 Kelly CR, Ihunnah C, Fischer M et al. Fecal microbiota transplant for treatment of Clostridium difficile infection in immunocompromised patients. Am J Gastroenterol 2014;109:1065-71.

21 Hamilton MJ, Weingarden AR, Sadowsky MJ, Khoruts A. Standardized frozen preparation for transplantation of fecal microbiota for recurrent Clostridium difficile infection. Am J Gastroenterol 2012;107:761-7.

22 Wilcox MH, Gerding DN, Poxton IR et al. Bezlotoxumab for prevention of recurrent Clostridium difficile infection. N Engl ] Med 2017:376:305-17

23 Gerding DN, Meyer T, Lee C et al. Administration of spores of nontoxigenic Clostridium difficile strain M3 for prevention of recurrent C. difficile infection. JAMA 2015;313:1719-27.

Address for correspondence: Dr Benjamin H Mullish, Division of Integrative Systems Medicine and Digestive Disease, 10th Floor, QEQM Wing, St Mary's Hospital Campus, Imperial College London, South Wharf Road, Paddington, London W2 1NY, UK. Email: b.mullish@imperial.ac.uk 